\title{
结构化矩阵优化的高光谱图像噪声去除算法
}

\author{
徐宏辉，郑建炜，秦梦洁，陈婉君 \\ (浙江工业大学计算机科学与技术学院 杭州 310023) \\ (zjw@zjut.edu.cn)
}

\begin{abstract}
摘 要: 受带噪线路或电子感应设备老化等影响, 高光谱图像在编码和传输过程中往往会被混合噪声污染, 严重影 响后续图像检测、分类、跟踪、解卷等应用的性能. 为实现有效地去噪，将零化滤波技术扩展至高光谱图像修复中，提 出一种结构化矩阵恢复的混合噪声去除算法. 首先根据高光谱图像不同波段之间的关联性和局部空间邻域的关滑性, 将不同图像子块构建成具有 Hankel 结构的低秩矩阵; 然后考虑 Hankel 化线性操作并不破坏混合噪声的稀疏状态, 将 稀疏性约束作为先验条件; 最后使用截断核范数和组稀疏范数分别替代低秩和稀疏约束函数, 构建双先验条件下的 目标模型, 并采用交替方向乘子法进行变量优化求解. 整体去噪流程通过图像 patch 分组、子块优化和 patch 重组 3 个步骤实现. 通过多组行业通用高光谱数据进行实验的结果表明，该算法在视觉效果和定量评价 PSNR, SSIM 以及 SAD 上都明显优于现有的高光谱噪声去除算法.
\end{abstract}

关键词：高光谱图像; 图像去噪; 结构化矩阵; 截断核范数; 交替方向乘子法

中图法分类号: TP391.41 DOI: 10.3724/SP.J.1089.2021.18159

\section{Hyperspectral Image Denoising Using Structural Matrix Optimization}

\author{
Xu Honghui, Zheng Jianwei", Qin Mengjie, and Chen Wanjun \\ (School of Computer Science and Technology, Zhejiang University of Technology, Hangzhou 310023)
}

\begin{abstract}
Due to various factors, e.g., thermal electronics, dark current, and stochastic error of photocounting in an imaging process, hyperspectral images (HSI) are inevitably corrupted by different types of noise during the acquisition and transmission process. For effective noise removal, in this paper, we attempt to extend the annihilating filter for the HSI community. Specifically, a new method based on the structural matrix recovery is presented. First, benefiting from the correlation of different spectral bands and the smoothness of local spatial neighborhood, the image patches are hankelized to be a structural low-rank matrix; Then considering that the linear hankelization does not destroy the sparse attribution of the impulse noise, sparsity prior can be employed as a constraint; Finally, by using the truncated nuclear norm and group sparse norm as the surrogates of the original low-rank and sparse function, our final model is formed by two prior conditions and be solved via the well-known ADMM optimization algorithm. The whole image denoising procedure involves three main steps, i.e., the decomposition of input image into overlapped patches, the block-wise estimates for each patch, and the recovery of the whole image. The experimental results show that our proposed method is superior to other state-of-the-art methods both visually and quantitative indices, such as peak signal to noise ratio (PSNR), structural similarity index measure (SSIM) and spectral angle distance (SAD).
\end{abstract}

收稿日期：2019-11-20；修回日期：2020-11-26. 基金项目：国家自然科学基金(61602413); 浙江省自然科学基金(LY19F030016); 浙江省教育厅科研项目(Y201941027). 徐宏辉(1996-), 男, 博士研究生, 主要研究方向为图像处理; 郑建炜(1982一), 男, 博士, 副 教授, 博士生导师, 论文通讯作者, 主要研究方向为机器学习、图像处理、数据分析; 秦梦洁(1995一), 女, 博士研究生, 主要研究方 向为图像处理; 陈婉君(1982一), 女, 硕士, 讲师, 主要研究方向为机器学习、数据分析. 
Key words: hyperspectral image; image denoising; structural matrix; truncated nuclear norm; alternating direction method of multiplier

高光谱图像(hyperspectral image, HSI)将光谱 技术和成像技术结合，不仅能够获取目标的空间 特征信息，而且能对每个空间像元经过色散形成 的几十乃至几百个窄波段进行连续的光谱覆盖, 从而获取目标的光谱特征. 高光谱遥感技术的出 现，使得基于图像和光谱的对地观测应用朝更加 精细准确的方向发展. 目前, 遥感技术已经广泛应 用于农业遥感、环境保护、地图测绘等诸多领域 ${ }^{[1]}$, 高光谱技术在社会生活和经济建设中发挥着越来 越重要的作用. 在具体应用过程中, 遥感图像在采 集、转换、传输、压缩和储存等实施步骤常常会因 成像设备或外部环境因素的影响而产生混合噪声 ${ }^{[2]}$. 例如, 图像传感器在拍摄时, 由于亮度不够均匀或 长时间工作使传感器温度过高, 会造成高斯噪声; 图像在编码和传输过程中经过含噪声的线路或者 被电子感应器噪声所污染, 会导致脉冲(椒盐)噪声 和条带丢失现象. 噪声的存在不仅影响高光谱遥 感图像的视觉效果, 而且在城市规划、测绘以及农 业作物分类和识别等方面的应用也受到严重影响. 所以，在保留图像原有基本信息的同时，有效地去 除 HSI 中的混合噪声已成为后续应用前的一个必 要的预处理步骤.

传统的去噪算法将 HSI 的数百个光谱通道逐 波段按照灰度图像进行处理. 如 $K$ 奇异值分解 ( $K$-singular value decomposition, $K$-SVD) ${ }^{[3]}$ 、块匹配 三维滤波(block matching and 3D filtering, BM3D $)^{[4]}$ 等算法对 HSI 数据进行简单的逐波段去噪处理, 但其处理过程忽略了不同光谱波段之间的相关性, 极大地限制了最后的恢复效果. 为引人这种相关 性先验条件, Zhong 等 ${ }^{[5]}$ 设计了一种多光谱带条件 随机场算法，可以在统一的概率框架内同时建模， 并利用空间和光谱依赖关系. Zhou 等 ${ }^{[6]}$ 提出一种基 于非参数贝叶斯字典学习(nonparametric Bayesian dictionary learning, BPFA)的高光谱遥感图像修复 算法. Shen 等 ${ }^{[7]}$ 在 BPFA 的基础上，进一步提出一 种自适应谱加权稀疏贝叶斯字典学习算法，在压 缩感知框架下，通过使用 BPFA 自适应地利用不同 光谱通道之间潜在的频谱相关性, 解决 HSI 修复 问题. 基于偏微分方程(partial differential equation, PDE)的算法在 HSI 处理中也得到了广泛的应用. 由于相邻的 HSI 波段之间通常表现出很强的关联 性, 通过将每个波段视为一个向量进行重组, 并将
所有向量叠加到一个矩阵中, 可以得到具有低秩 特性的目标变量. 基于此, 采用低秩矩阵近似 (low-rank matrix approximation, LRMA)的 HSI 恢复 算法得到了广泛的应用。受鲁棒主成分分析 (robust principal component analysis, RPCA ${ }^{[8]}$ 思想 的启发，Xie 等 ${ }^{[9]}$ 将 HSI 分成多个重叠的 patch，并 按字典顺序排列成二维矩阵, 从而将噪声建模为 稀疏成分，最后依 LRMA算法优化每个 patch 元素， 进行整体 HSI 的排列去噪. 为了更有效地对空间 光谱相似像素进行分组, $\mathrm{Lu}$ 等 ${ }^{[10]}$ 引人了一种空间 自适应局部相似像素搜索算法，使所选择的 patch 分组具有更可靠的邻接关系.

针对 HSI 不同频段的噪声强度各向异性, 文 献[11]提出了一种基于随机 SVD 的噪声调整迭代 LRMA 算法. Chen 等 ${ }^{[12]}$ 将 HSI 转化为低秩 Casorati 矩阵, 以充分利用谱相关性对其进行矩阵分解, 以 避免 SVD 的复杂运算, 同时引人 Framelet 正则项构 建基于 Framelet 的低秩非负矩阵框架 (Frameletregularized low-rank nonnegative matrix factorization, LRNMF)进行去噪. $\mathrm{He}$ 等 ${ }^{[13]}$ 将 HSI 分成多个 patch 并将其转换成低秩 Casorati 矩阵, 再将所有 patch 进行联合优化，构建空间光谱全局低秩矩阵恢复模 型(low-rank matrix recovery and global spatial-spectral total variation, LLRGTV), 以探索 HSI 的局部低秩 特性和全局空间光谱性, 从而对 HSI 进行去噪. Chen 等 ${ }^{[14]}$ 利用光谱间的相关性，提出加权组稀疏 全变分低秩张量(weighted group sparsity-regularized low-rank tensor decomposition, LRTDGS)模型对图 像进行去噪. Zheng 等 ${ }^{[15]}$ 将 HSI 进行张量分解, 并 对分解的 2 项因子进行约束优化, 提出了双因子正 则化低秩张量分解(double-factor-regularized low-rank tensor factorization, LRTF-DFR)算法，对图像实施 去噪. 上述算法都是使用矩阵和张量本身数据直 接进行处理, 继而通过低秩和邻域相似性等先验 条件构建目标模型. 然而, 这些先验特性在空间域 往往仅处于近似状态 ${ }^{[16]}$, 将其作为绝对约束准则 只能得到目标的次优解. 因此, 将 HSI 变换至特征 结构矩阵(例如, Toeplitz 矩阵, Hankel 矩阵等), 挖 掘本质的先验约束条件, 并添加至去噪、超解析等 模型成为一个新兴的研究方向.

近年来, 部分研究者根据有限新息率(finite rate 
of innovations, FRI $)^{[17]}$ 采样理论, 将信号处理中的 零化滤波(annihilating filter, $\mathrm{AF}$ ) 技术引人图像处理 领域，构造面向图像的结构化矩阵，并提出新的修 复算法. 这类算法利用图像谱成分稀疏所引起的 傅里叶系数与像素结构之间的零化关系, 通过 $\mathrm{AF}$ 构建卷积关联矩阵, 然后将图像修复问题转化为 结构矩阵填充问题. 例如, Ongie 等 ${ }^{[18]}$ 将医学图像 的傅里叶系数构建成 Toeplitz 矩阵用于修复图像. LORAKS 框架 ${ }^{[19]}$ 利用连续域图像的离散域稀疏性 和平滑相位构建出结构化的低秩矩阵, 其比传统 的 $l_{1}$ 恢复策略具有更加平滑的效果. Jin 等 ${ }^{[20]}$ 将所述 结构矩阵移植于自然图像的修复应用. Yokota 等 ${ }^{[21]}$ 将结构矩阵进行张量化处理，在条状缺失的自然 图像修复中取得了突出的性能.

现存结构化优化算法将 $\mathrm{AF}$ 技术运用于矩阵或 张量填充问题, 在自然图像修复、磁共振图超解析 等方面获得了成功的应用. 然而，上述算法仍存在 若干共性问题: 首先，直接通过零化滤波器构建结 构化矩阵并约束目标函数, 缺乏理论依据和可行 性保证; 其次，单纯的低秩约束不足以支撑具体应 用问题中复杂的先验分布状态. 针对这些问题，本 文结合空间域 patch 操作 ${ }^{[9]}$ 和结构化矩阵的优势, 将 AF 技术扩展至 HSI 去噪中

\section{$1 \quad \mathbf{A F}$ 技术}

\subsection{FRI 恢复理论}

由于一般图像都具备局部光滑性，本节以分 段常数的一阶信号为例描述基本的 FRI 恢复框架. 不失一般性，给定变量域为 $[0,1]$ 的信号

$$
x(t)=\sum_{i=1}^{K-1} a_{i} 1\left[t_{i}, t_{i+1}\right](t)
$$

其中, $a_{i}$ 为信号幅值; $0<t_{1}<t_{2}<\cdots<t_{K}<1$ 是边界位 置; $1_{U}$ 则是集合 $U$ 中的特征函数. $x(t)$ 的低通傅里 叶系数为

$$
\hat{x}[k]=\int_{0}^{1} x(t) \mathrm{e}^{-\mathrm{j} 2 \pi k t} \mathrm{~d} t, k \in \mathbb{Z},|k| \leqslant N
$$

依式(1)可知, 微分 $\partial x$ 由 $K$ 阶狄拉克流组成, 即

$$
\partial x(t)=\sum_{i=1}^{K} c_{i} \delta\left(t-t_{i}\right)
$$

其中, $c_{i}=a_{i}-a_{i-1}, i=1, \cdots, K$ 且 $a_{K}=a_{0}=0$. 根据文 献[17]可知, 该信号有 $2 K$ 个自由度, 即振幅 $\left\{c_{i}\right\}_{i=1}^{K}$ 和 $\left\{t_{i}\right\}_{i=1}^{K}$, 其傅里叶系数是 $K$ 个指数的线性组合, 即

$$
\widehat{\partial x}(k)=\sum_{i=1}^{K} c_{i} \mathrm{e}^{-\mathrm{j} 2 \pi k t_{i}}
$$

具体计算可由已知的 $\hat{x}[k]$ 通过傅里叶域关系 $\widehat{\partial x}(k)=\mathrm{j} 2 \pi k \hat{x}(k)$ 实现. 因此, 信号 $x$ 的恢复问题简 化为通过已知的 $2 N+1$ 个样本估计 $2 K$ 个未知参数 $\left\{c_{i}, t_{i}\right\}_{i=1}^{K}$, 其解通过所谓的零化多项式获得.

定义零化多项式为

$$
\mu(t)=\prod_{i=1}^{K}\left(e^{\mathrm{j} 2 \pi t}-e^{\mathrm{j} 2 \pi t_{i}}\right)=\sum_{k=0}^{K} c[k] e^{\mathrm{j} 2 \pi k t}
$$

其零值位于且仅位于边界位置 $\left\{t_{i}\right\}_{i=1}^{K}$. 由于傅里叶 系数 $\widehat{\partial x}(k)$ 仅在边界位置取非零值, 因此其可通过 卷积操作被系数 $c[k]$ 零化, 即

$$
\sum_{k=0}^{K} c[k] \widehat{\partial x}[l-k]=0
$$

其中, $l \in \mathbb{Z}$. 由式(6)进一步可知, 在空间域的零 化关系为 $\mu \partial x=0$.

综上, 假设已知至少 $2 K$ 个连续 $\widehat{\partial x}$ 样本 $(N \geqslant K)$, 则可依式(6)构建一个线性 Toeplitz 系统 并求解 $K+1$ 个未知系数 $c[k]$. 进一步, 边界位置 $\left\{t_{i}\right\}_{i=1}^{K}$ 可由 $\mu(t)$ 的根计算得到; 最后, 可通过将 $\left\{t_{i}\right\}_{i=1}^{K}$ 代人式(4)并求解该过完备线性方程获取未 知系数 $c_{i}$, 进而推演出幅值 $a_{i}$.

\section{2 结构化矩阵构建}

由第 1.1 节可知, $c[k]$ 是长度为 $K+1$ 的 $\mathrm{AF}$ 系 数. 实际上, 可进一步找到长度为 $k_{1}$ 的滤波器 $w[k]$, 用其将 $c[k]$ 扩展为 $\hat{h}_{w}[k]$, 其中

$$
\hat{h}_{w}[k]=(w * c)[k]
$$

由式(6)可得 $(c * \widehat{\partial x})[k]=0$, 则有 $(w * c * \widehat{\partial x})[k]=$ $\left(\hat{h}_{w} * \widehat{\partial x}\right)[k]=0$. 因此 $\hat{h}_{w}[k]$ 也满足式(6)关系, 即也 可称 $\hat{h}_{w}[k]$ 为零化滤波器, 使得

$$
\left(\hat{h}_{w} * \widehat{\partial x}\right)[k]=\sum_{k=0}^{K} \hat{h}_{w}[k] \widehat{\partial x}[l-k]=0, \forall k
$$

式(8)可以表示为矩阵形式

$$
\boldsymbol{H}_{1}(\boldsymbol{X}) \hat{\boldsymbol{h}}_{\boldsymbol{w}}=0
$$

其中, $\boldsymbol{H}_{1}(\boldsymbol{X})$ 是具有 Hankel 结构的矩阵, 其形式为

$\left[\begin{array}{ccccc}\widehat{\partial x}[0] & \widehat{\partial x}[1] & \widehat{\partial x}[2] & \cdots & \widehat{\partial x}[d-1] \\ \widehat{\partial x}[1] & \widehat{\partial x}[2] & \widehat{\partial x}[3] & \ldots & \widehat{\partial x}[d] \\ \vdots & \vdots & \vdots & \ddots & \vdots \\ \widehat{\partial x}[n-d] & \widehat{\partial x}[n-d+1] & \widehat{\partial x}[n-d+2] & \cdots & \widehat{\partial x}[n-1]\end{array}\right]$.

其中, $n$ 为矩阵束; $d=K+k_{1} ; \hat{\boldsymbol{h}}_{w}$ 的形式描述为

$$
\hat{\boldsymbol{h}}_{\boldsymbol{w}}=\left[\hat{h}_{w}[d-1], \hat{h}_{w}[d-2], \cdots, \hat{h}_{w}[0]\right]^{\mathrm{T}}
$$




\section{2 算法提出与优化}

\subsection{HSI 的 Hankel 矩阵构建}

与一维分段常数信号类似, HSI 的空间域亦具 备局部光滑特性. 根据文献[9]可知, 将 HSI 进行 可重叠的区域划分，所得的 patch 子块具有明显的 像素集聚度, 则可将其视为二维分段函数, 即

$$
x(r)=\sum_{i=1}^{K-1} a_{i} 1_{U_{i}}(r), r=(v, \kappa) \in[0,1]^{2}
$$

其中, $a_{i}$ 为信号幅值; $1_{U}$ 则是集合 $U$ 中的特征函数; $U_{i} \in[0,1]^{2}$, 其边缘集为 $R=\bigcup_{i=1}^{N} \partial U_{i}$. 类似于第 1.1 节 描述, 二维分段光滑函数的频谱可建模为二维 $K$ 阶 狄拉克流

$$
\widehat{x}(\boldsymbol{\omega})=\sum_{i=1}^{K} c_{i} \delta\left(\boldsymbol{\omega}-\boldsymbol{\omega}_{i}\right), \boldsymbol{\omega}=\left(\omega_{v}, \omega_{\kappa}\right)
$$

同样，二维分段光滑函数也可通过有限采样 点进行稀疏傅里叶系数重构, 但分段函数具有无 限的自由度. 因此, 在无约束情形下恢复式(11)所 述信号几乎是不可能的. 文献[16]将信号边缘集限 制为二维带限周期函数的零值集合, 则可采用零 化滤波器 $\widehat{\boldsymbol{h}}(\boldsymbol{\omega})$ 恢复式(12), 即

$$
\widehat{\boldsymbol{h}}(\boldsymbol{\omega}) \widehat{x}(\boldsymbol{\omega})=0, \forall \boldsymbol{\omega}
$$

上述 $\widehat{\boldsymbol{h}}(\boldsymbol{\omega})$ 为频率中的零化滤波器, 因此在图像域 中存在相对应的零化滤波器 $\boldsymbol{h}(r):=\mathcal{F}^{-1} \widehat{\boldsymbol{h}}(\boldsymbol{\omega})$, 使得

$$
\boldsymbol{h}(r) * x(r)=0
$$

其中, $\mathcal{F}^{-1}(\cdot)$ 为傅里叶逆变换.

通过以上分析，给定 HSI 大小为 $m \times n$ 的 patch, 可以相应找到一个 $p \times q$ 的零化滤波器 $c\left[k_{v}, k_{\kappa}\right]$, 使得

$$
(c * x)\left[k_{v}, k_{\kappa}\right]=0
$$

式(15)可以进一步表示为

$$
\boldsymbol{H}(\boldsymbol{X}) \boldsymbol{c}=0
$$

其中，

$$
\boldsymbol{H}(\boldsymbol{X})=\left[\begin{array}{cccc}
\boldsymbol{H}\left(x_{0}\right) & \boldsymbol{H}\left(x_{1}\right) & \cdots & \boldsymbol{H}\left(x_{q-1}\right) \\
\boldsymbol{H}\left(x_{1}\right) & \boldsymbol{H}\left(x_{2}\right) & \cdots & \boldsymbol{H}\left(x_{q}\right) \\
\vdots & \vdots & \ddots & \vdots \\
\boldsymbol{H}\left(x_{n-q}\right) & \boldsymbol{H}\left(x_{n-q+1}\right) & \cdots & \boldsymbol{H}\left(x_{n-1}\right)
\end{array}\right]
$$

是具有 Hankel 结构的二维矩阵. 其中, $\boldsymbol{H}(\boldsymbol{X}) \in$ $\mathbb{R}^{(n-q+1) \times q}$ 和 $\boldsymbol{H}\left(x_{j}\right) \in \mathbb{R}^{(m-p+1) \times p}$ 同样为 Hankel 结构 矩阵, 具体形式为

$\left[\begin{array}{ccccc}x[0, j] & x[1, j] & x[2, j] & \cdots & x[p-1, j] \\ x[1, j] & x[2, j] & x[3, j] & \cdots & x[p, j] \\ \vdots & \vdots & \vdots & \ddots & \vdots \\ x[m-p, j] & x[m-p+1, j] & x[m-p+2, j] & \cdots & x[m-1, j]\end{array}\right]$.

以 patch 大小 $5 \times 5$ 为例, 具体 $\boldsymbol{H}(\boldsymbol{X})$ 构建方式及 其与所采用的高光谱图 patch 关系如图 1 所示.

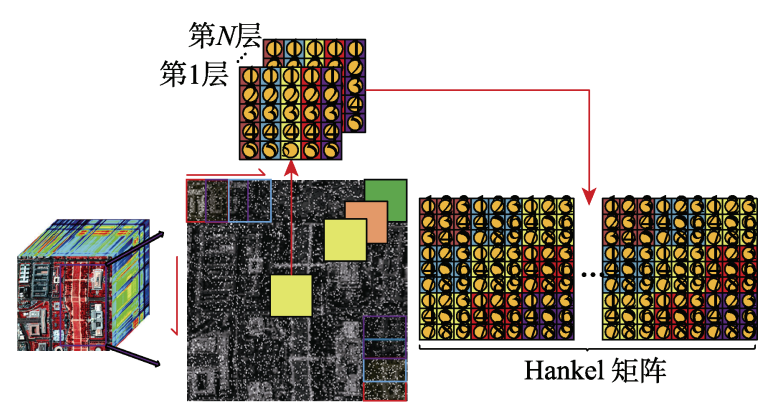

图 $1 \boldsymbol{H}(\boldsymbol{X})$ 的组成以及所采用的 patch 结构示意

首先，将 HSI 分成多个子 patch，每一个 patch 包含 HSI 的所有通道(图 1 以显示 2 层为例). 然后, 在每一层中, 将任意 patch 不同列标注为 5 个不同 颜色, 所有列向量经过 $\mathrm{AF}$ 卷积操作, Hankel 转化 为一个 $3 \times 3$ 的块结构化矩阵, 其中每个子块矩阵 又以 Hankel 结构展开; 同时所有的通道依次并排 展开组成一个大 Hankel 矩阵. 针对所有通道实施 上述结构化操作, 即可依式(17)将所有通道 patch 转换为最终目标矩阵.

\subsection{Hankel 矩阵的低秩性}

众所周知，原始高光谱子 patch 的空间结构具 有近似低秩性 ${ }^{[9]}$, 而式(17)构造了二维 Hankel 矩 阵, 本节证明其具有绝对低秩性, 为后续低秩约束 的目标函数构建提供理论依据. 考虑 Hankel 结构 的一致性, 仅以一维信号 Hankel 矩阵为例进行分 析描述. 首先, 可将零化滤波器重新表示为

$$
\tilde{\hat{h}}_{w}=Q w
$$

其中, $\tilde{\hat{\boldsymbol{h}}}_{\boldsymbol{w}}$ 是 $\hat{\boldsymbol{h}}_{w}$ 的逆序排列向量, 具体形式为

$$
\tilde{\hat{\boldsymbol{h}}}_{w}=\left[\hat{h}_{w}[0], \hat{h}_{w}[1], \cdots, \hat{h}_{w}[d-1]\right]^{\mathrm{T}}
$$

向量 $\boldsymbol{w}$ 则可写为

$$
\begin{gathered}
\boldsymbol{w}=\left[w[0], w[1], \cdots, w\left[k_{1}-1\right]\right]^{\mathrm{T}} \\
\boldsymbol{Q}=\left[\begin{array}{cccc}
c[0] & 0 & \cdots & 0 \\
c[1] & c[0] & \cdots & 0 \\
\vdots & \vdots & \ddots & \vdots \\
c[K] & c[K-1] & \cdots & c\left[K-k_{1}-1\right] \\
\vdots & \vdots & \ddots & \vdots \\
0 & 0 & \cdots & c[K]
\end{array}\right]
\end{gathered}
$$


是通过 $c[k]$ 构造的矩阵.

由式(21)可知, $\boldsymbol{Q}$ 是满秩矩阵，且向量 $\boldsymbol{w}$ 是非 零向量. 由此 $\tilde{\hat{\boldsymbol{h}}}_{w}$ 也为非零向量, 为矩阵 $\boldsymbol{Q}$ 的值域 空间(range space). 此外, 由式(9)可得 $\hat{\boldsymbol{h}}_{\boldsymbol{w}}$ 是 $\boldsymbol{H}_{1}(\boldsymbol{X})$ 的零空间 ${ }^{[22]}$. 因此，条件 $\hat{\boldsymbol{h}}_{w} \neq 0$ 表明 $\boldsymbol{Q}$ 的值域空 间即为 $H_{1}(X)$ 的零空间. 换言之, $H_{1}(X)$ 存在非 零解. 根据文献[22]， $\boldsymbol{H}_{1}(\boldsymbol{X})$ 的列空间不满秩，即 该矩阵的列向量存在线性相关性, 因此 $\boldsymbol{H}_{1}(\boldsymbol{X})$ 是 低秩的，即

$$
\operatorname{rank}\left(\boldsymbol{H}_{1}(\boldsymbol{X})\right) \leqslant K
$$

其中, $\operatorname{rank}(\cdot)$ 表示矩阵的秩运算.

\subsection{Hankel 结构化矩阵的 HSI 混合噪声去除 算法}

在混合噪声干扰下，结合噪声的稀疏性和 patch 的近似低秩性, HSI 去噪问题可以由常规 LRMA 模型求解. Shen 等 ${ }^{[23]}$ 将 LRMA 理想化为一个 鲁棒主成分分析 ${ }^{[8]}$ 问题. 假设低秩矩阵 $\boldsymbol{A} \in \mathbb{R}^{m \times n}$ 被 稀疏误差矩阵 $\boldsymbol{S} \in \mathbb{R}^{m \times n}$ 破坏，则观测到的数据矩 阵 $\boldsymbol{M} \in \mathbb{R}^{m \times n}$ 可以分解为稀疏矩阵与低秩矩阵的和, 即 $\boldsymbol{M}=\boldsymbol{A}+\boldsymbol{S}$. 理想的 RPCA 可以描述为从已知的 观测数据矩阵 $\boldsymbol{M}$ 恢复出低秩矩阵 $\boldsymbol{A}$ 和稀疏误差矩 阵 $\boldsymbol{S}$. 该优化问题的公式为

$$
\min _{\boldsymbol{A}, \boldsymbol{S}} \operatorname{rank}(\boldsymbol{A})+\lambda\|\boldsymbol{S}\|_{0}, \text { s.t. } \boldsymbol{M}=\boldsymbol{A}+\boldsymbol{S}
$$

其中, \|\|$_{0}$ 表示 $l_{0}$ 范数; $\lambda$ 表示 2 项约束间的平衡 参数.

式(23)是一个 NP 难问题，无法得到有效的解 方案. 针对该难题，文献[24-25]分别采用 $l_{1}$ 范数和 核范数代替 $l_{0}$ 范数和秩函数, 以获得有效的凸优 化解. 具体描述为

$$
\min _{\boldsymbol{A}, \boldsymbol{S}}\|\boldsymbol{A}\|_{*}+\lambda\|\boldsymbol{S}\|_{1} \text {, s.t. } \boldsymbol{M}=\boldsymbol{A}+\boldsymbol{S}
$$

其中, $\|\boldsymbol{A}\|_{*}=\sum_{i} \sigma_{i}(\boldsymbol{A})$ 是 $\boldsymbol{L}$ 的核范数, $\sigma_{i}(\boldsymbol{A})$ 是矩阵 $\boldsymbol{A}$ 的奇异值.

根据第 2.1 节和第 2.2 节描述, Hankel 结构化 变换后的矩阵较原始高光谱 patch 具有更为绝对的 低秩性，可替换式(24)的低秩约束项. 此外, Hankel 矩阵仅针对图像元素实施线性操作, 因此变换过 程并不影响混合噪声的稀疏性. 最后, 本课题组前 期工作 ${ }^{[26]}$ 已证明, 截断核范数较经典的核范数约 束具有更好的性能. 结合上述观点，本文提出基于 截断核范数的低秩 Hankel 矩阵恢复算法(low-rank hankel matrix recovery based on truncated nuclear norm, HTLRMA), 其目标函数描述为

$$
\min _{\boldsymbol{X}, \boldsymbol{S}}\|\boldsymbol{H}(\boldsymbol{X})\|_{r}+\lambda\|\boldsymbol{S}\|_{1} \text {, s.t. }\|\boldsymbol{M}-\boldsymbol{H}(\boldsymbol{X})-\boldsymbol{S}\|<\varepsilon
$$

其中, $\|\boldsymbol{H}(\boldsymbol{X})\|_{r}=\sum_{i=r}^{\min (m-p+1, p)} \sigma_{i}(\boldsymbol{H}(\boldsymbol{X}))$ 是截断核范 数的定义; $r$ 是截断参数; $\varepsilon$ 表示混合噪声中高斯成 分的方差. 详细的截断核范数性质请参考文献[26] 相关内容. 值得注意的是, 式(25)延用了经典的变 量表示形式 $\boldsymbol{M}$ 和 $\boldsymbol{S}$, 仅将其矩阵尺寸调整至 Hankel 化后的规模.

\subsection{HTLRMA 模型求解}

采用经典的交替方向乘子法(alternating direction method of multipliers, ADMM) $)^{[27]}$ 优化求解 HTLRMA 模型中的未知变量 $\boldsymbol{H}(\boldsymbol{X})$ 和 $\boldsymbol{S}$. 首先, 将 式(25)重写为增广拉格朗日函数形式

$$
\begin{aligned}
& \mathcal{J}(\boldsymbol{H}(\boldsymbol{X}), \boldsymbol{S}, \boldsymbol{Z})=\|\boldsymbol{H}(\boldsymbol{X})\|_{r}+\lambda\|\boldsymbol{S}\|_{1}+ \\
& \langle\boldsymbol{Z}, \boldsymbol{M}-\boldsymbol{H}(\boldsymbol{X})-\boldsymbol{S}\rangle+\frac{\rho}{2}\|\boldsymbol{M}-\boldsymbol{H}(\boldsymbol{X})-\boldsymbol{S}\|_{\mathrm{F}}^{2}
\end{aligned}
$$

其中, $\boldsymbol{Z}$ 为拉格朗日乘子; $\rho$ 是惩罚参数; $\langle\cdot\rangle$ 表示 内积运算; \|\|$_{\mathrm{F}}$ 为 $\mathrm{F}$ 范数约束.

根据 ADMM 的规则, 式(26)可以通过固定余 下变量逐个求解各未知量进行优化. 在第 $L$ 次迭 代中，分别假设 $\boldsymbol{H}^{L}(\boldsymbol{X}), \boldsymbol{S}^{L}$ 和 $\boldsymbol{Z}^{L}$ 未知，目标问题 可分为 3 个子问题, 即求解 $\boldsymbol{H}^{L+1}(\boldsymbol{X}), \boldsymbol{S}^{L+1}$ 和 $\boldsymbol{Z}^{L+1}$. 各子问题的具体形式为

$$
\begin{gathered}
\boldsymbol{H}^{L+1}(\boldsymbol{X})=\underset{\boldsymbol{H}(\boldsymbol{X})}{\operatorname{argmin}} \mathcal{J}\left(\boldsymbol{H}(\boldsymbol{X}), \boldsymbol{S}^{L}, \boldsymbol{Z}^{L}\right)= \\
\underset{\boldsymbol{H}(\boldsymbol{X})}{\arg \min } \frac{1}{\rho}\|\boldsymbol{H}(\boldsymbol{X})\|_{r}+\frac{1}{2}\left\|\boldsymbol{H}(\boldsymbol{X})+\boldsymbol{S}^{L}-\boldsymbol{M}-\boldsymbol{Z}^{L} / \rho\right\|_{\mathrm{F}}^{2} \\
\boldsymbol{S}^{L+1}=\underset{\boldsymbol{S}}{\arg \min } \mathcal{J}\left(\boldsymbol{H}^{L+1}(\boldsymbol{X}), \boldsymbol{S}, \boldsymbol{Z}^{L}\right)= \\
\underset{\boldsymbol{S}}{\arg \min } \lambda \rho^{-1}\|\boldsymbol{S}\|_{1}+ \\
\frac{\rho}{2}\left\|\boldsymbol{S}+\boldsymbol{H}^{L+1}(\boldsymbol{X})-\boldsymbol{M}+\rho^{-1} \boldsymbol{Z}^{L}\right\|_{\mathrm{F}}^{2} \\
\boldsymbol{Z}^{L+1}=\boldsymbol{Z}^{L}+\rho\left(\boldsymbol{H}^{L+1}(\boldsymbol{X})+\boldsymbol{S}^{L+1}-\boldsymbol{M}\right)
\end{gathered}
$$

各变量的具体求解过程描述如下.

(1) 求解 $\boldsymbol{H}(\boldsymbol{X})$ 子问题. 由于截断核范数带 来的非凸松驰, 传统的奇异值阈值算法不能对 其有效求解, 因此本文采用部分奇异值阈值操 作 ${ }^{[28]}$ 进行求解. 定义 $\boldsymbol{Y}=\boldsymbol{M}-\boldsymbol{S}^{L}+\boldsymbol{Z}^{L} / \rho$, 并令 $\varsigma=\min (m-q+1, q)$, 则 得 到 $\boldsymbol{H}(\boldsymbol{X})$ 的解为 $P_{1 / \rho}(\boldsymbol{Y})=\boldsymbol{U}_{\boldsymbol{Y}}\left(\boldsymbol{D}_{\boldsymbol{Y}_{1}}+\boldsymbol{S}_{1 / \rho}\left[\boldsymbol{D}_{\boldsymbol{Y}_{2}}\right]\right) \boldsymbol{V}_{\boldsymbol{Y}}^{\mathrm{T}}$, 其中, $\boldsymbol{S}_{1 / \rho}[x]=$

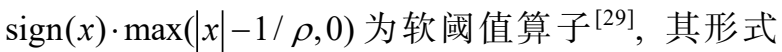


为 $S_{1 / \rho}[x]=\operatorname{sign}(x) \cdot \max (|x|-1 / \rho, 0), \boldsymbol{U}_{\boldsymbol{Y}}$ 和 $\boldsymbol{V}_{\boldsymbol{Y}}$ 分别 是 $\boldsymbol{Y}$ 的左右奇异值向量矩阵, $\boldsymbol{D}_{\boldsymbol{Y}_{1}}=\operatorname{diag}\left(\sigma_{1}, \cdots, \sigma_{r}, \mathbf{0}\right), \boldsymbol{D}_{\boldsymbol{Y}_{2}}=\operatorname{diag}\left(\mathbf{0}, \sigma_{r} \cdots, \sigma_{\varsigma}\right), \mathbf{0}$ 则 表示合适长度的零向量.

(2) 求解 $\boldsymbol{S}$ 子问题. $\boldsymbol{S}$ 子问题可直接使用软 阈值收缩算子来获得闭式解, 其解为

$$
\boldsymbol{S}^{L+1}=\boldsymbol{S}_{\lambda / \rho}\left(\boldsymbol{M}-\boldsymbol{H}^{L+1}(\boldsymbol{X})+\rho^{-1} \boldsymbol{Z}^{L}\right)
$$

其中, $\boldsymbol{S}_{\lambda / \rho}[x]$ 为软阈值算子 ${ }^{[29]}$, 形式为 $\boldsymbol{S}_{\lambda / \rho}[x]=$ $\operatorname{sign}(x) \cdot \max (|x|-\lambda / \rho, 0)$.

根据式(27) 式(29)这 3 个关键优化运算, 算 法基于 ADMM 的 HTLRMA 模型优化给出了 Hankel 子块矩阵的优化方案. 当收敛条件 $\left\|\boldsymbol{H}^{L+1}(\boldsymbol{X})-\boldsymbol{H}^{L}(\boldsymbol{X})\right\|_{\mathrm{F}}^{2} /\left\|\boldsymbol{H}^{L}(\boldsymbol{X})\right\|_{\mathrm{F}}^{2} \leqslant 10^{-4}$ 满足或迭 代次数达到 500 时令算法结束. 需要注意的是, 在 式(25)中, 高斯噪声方差由 $\varepsilon$ 表示, 其在 ADMM算 法的正则化操作式(26)中被替换为惩罚参数 $\rho$. 进 一步, 采用自适应规则依迭代次数增加而调整 $\rho$ 值, 即设置初始参数 $\rho_{0}=10^{-3}$, 并在第 $L$ 次迭代中 更新为 $\rho^{L+1}=\min \left(1.05 \rho^{L}, 10^{10}\right)$, 能够实现先挖掘 低秩和稀疏先验, 再抑制高斯噪声的分级优化操 作，又能加速 ADMM 的收玫效率.

算法 1. 基于 ADMM 的 HTLRMA 模型优化.

输人. 由各 patch 构建得到的 Hankel 矩阵, 约 束秩 $r, \lambda>0, \rho_{0}=10^{-3}$.

输出. $\boldsymbol{X}^{L+1}$ 和 $\boldsymbol{S}^{L+1}$.

Step1. 初始化. $\boldsymbol{H}^{0}(\boldsymbol{X})=\boldsymbol{S}=0, L=0$.

Step2. 令 $L=L+1$, 迭代 Step3 Step6, 直至收玫条 件满足.

Step3. $\boldsymbol{H}^{L+1}(\boldsymbol{X})=P_{1 / \rho}\left(\boldsymbol{M}-\boldsymbol{S}^{L}+\boldsymbol{Z}^{L} / \rho\right)$.

Step4. $\boldsymbol{S}^{L+1}=S_{\lambda / \rho}\left(\boldsymbol{M}-\boldsymbol{H}^{L+1}(\boldsymbol{X})+\rho^{-1} \boldsymbol{Z}^{L}\right)$.

Step5. $\boldsymbol{Z}^{L+1}=\boldsymbol{Z}^{L}+\rho\left(\boldsymbol{H}^{L+1}(\boldsymbol{X})+\boldsymbol{S}^{L+1}-\boldsymbol{M}\right)$.

Step6. $\rho^{L+1}=\min \left(1.05 \rho^{L}, 10^{10}\right)$.

Step7. 将 $\boldsymbol{H}^{L+1}(\boldsymbol{X})$ 逆 Hankel 化至图像 patch $\boldsymbol{X}^{L+1}$.

图 2 进一步给出了完整的 HSI 修复流程. 由于 图像局部邻域像素点往往具有明确的结构信息, 因此如图 2 中 $S_{1}$ 步骤所述的 patch 分块处理是一种 常见的视觉修复手段, 利用局部像素点强关联特 性挖掘低秩先验属性. 进一步, 为消除 patch 化处 理产生的边缘不连贯现象, 在分块操作中处理为 重叠 patch 结构, 将整体图像去噪问题转化为 $N$ 个 patch 去噪子问题, 其中 $N$ 由子块大小、移动步长 以及图像尺寸决定. $S_{2}$ 步骤用于将所有 patch 实施 Hankel 化操作, 即变换为具有绝对低秩性的结构
化矩阵, 由于该线性操作并不改变噪声的稀疏分 布，因此可以通过低秩和稀疏 2 个约束对子块进行 去噪, 模型如式(25)所示. 步骤 $S_{3}$ 通过算法 1 实现, 是修复工作的核心. 将算法 1 优化后的 Hankel 矩 阵由 $S_{4}$ 逆变换至原始 patch 矩阵, 完成单块去噪操 作. 值得注意的是, 需要在低秩和稀疏约束下遍历 所有子块, 完成 $N$ 次优化运算, 最后将所有 patch 合成为恢复所得的 HSI.

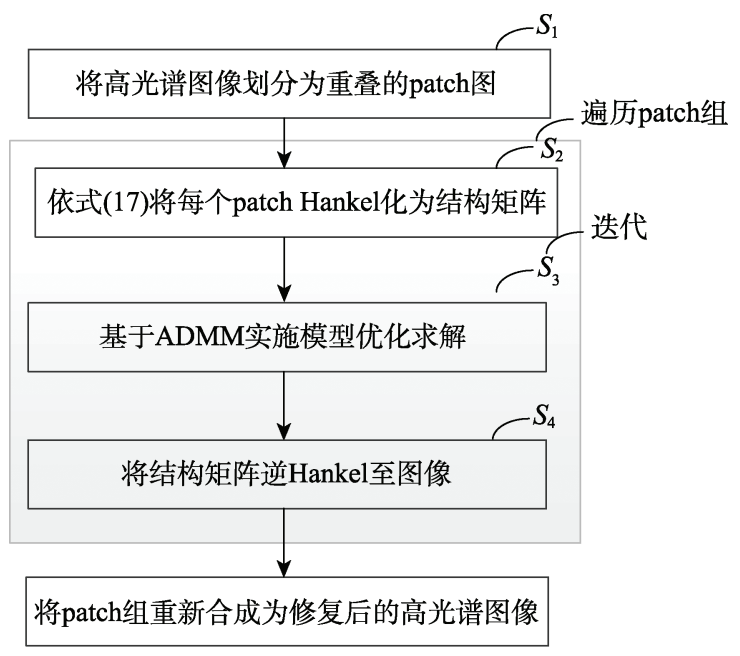

图 2 HSI 去噪流程

\section{3 实验结果与分析}

\section{1 算法性能对比}

为验证本文 HTLRMA 算法在 HSI 图像去除混 合噪声应用中的有效性, 选取 2 组规模各异的遥感 数据进行实验对比. 第 1 组数据是由高光谱数字图 像采集试验仪拍摄的 Washington D C Mall，其原始 空间尺寸为 $1208 \times 307 \times 191$ 像素，受运算效率限制， 实验中将该 HSI 图像缩减成 $256 \times 256 \times 191$, 如图 3a 所示. 第 2 组数据为 Pavia City Center, 其张量规模 为 $200 \times 200 \times 80$, 如图 $3 \mathrm{~b}$ 所示. 为了便于数值计算 和性能对比, 将 HSI 的所有波段像素值统一规范化 区间 $[0,1]$, 待算法重构后再逆操作至原始像素范 围. 对比算法为 5 种去噪模型 LRTV ${ }^{[30]}$, LRNMF ${ }^{[12]}$, LLRGTV $^{[13]}$, LRTDGS ${ }^{[14]}$ 以及 LRTF-DFR ${ }^{[15]}$. 其中, LRTV 对低秩性和光滑性进行了联合约束; LRNMF 构建基于 Framelet 的低秩非负矩阵框架; LLRGTV 针对局部 patch 实施低秩约束, 又对全局图像添加 了光滑性约束; LRTDGS 利用光谱间的相关性提出 加权组稀疏全变分低秩张量模型; LRTF-DFR 对图 像进行分解并对分解的 2 项因子进行约束优化, 所 采用的对比算法都在 HSI 图像去噪应用中被验证为 具有较强的鲁棒性和恢复能力. 


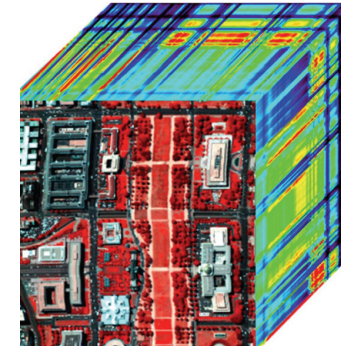

a. Washington D C Mall

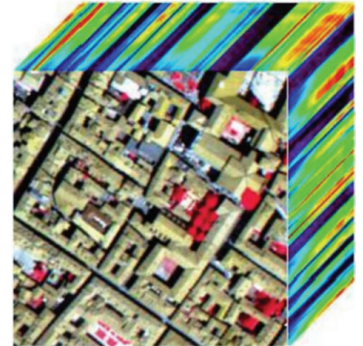

b. Pavia City Center
图 3 实验所采用的高光谱数据样本

为衡量各算法在混合噪声含量下的实施效果, 分别通过脉冲噪声、条带噪声以及高斯噪声污染所 采用的高光谱样本, 进行 4 种图像去噪实验. 同时 采用可视化效果和数值评价指标验证具体算法实 现的有效性，其中定量数值评价指标包括常用的 峰值信噪比(peak signal to noise ratio, PSNR) ${ }^{[13]}$ 指 数、结构相似度指数( structural similarity, SSIM) ${ }^{[31]}$ 和平均光谱角距离 (spectral angle distance, SAD $)^{[13]}$. 在操作过程中, 分别计算每一个波段的 PSNR 值、 SSIM 值以及 SAD 值，再对所有波段取均值，得到 PSNR 均值(MPSNR), SSIM 均值(MSSIM)以及 SAD 均值(MSAD), 它们分别定义为

$$
\begin{gathered}
\operatorname{PSNR}_{i}=10 \times \lg \frac{255^{2}}{\frac{1}{m n} \sum_{x=1}^{m} \sum_{y=1}^{n}\left[\boldsymbol{B}_{i}(x, y)-\boldsymbol{A}_{i}(x, y)\right]^{2}} \\
\operatorname{SSIM}_{i}=\frac{\left(2 \mu_{\boldsymbol{A}_{i}} \mu_{\boldsymbol{B}_{i}}+C_{1}\right)\left(2 \sigma_{\boldsymbol{A}_{i} \boldsymbol{B}_{i}}+C_{2}\right)}{\left(\mu_{\boldsymbol{A}_{i}}^{2}+\mu_{\boldsymbol{B}_{i}}^{2}+C_{1}\right)\left(\sigma_{\boldsymbol{A}_{i}}^{2}+\sigma_{\boldsymbol{B}_{i}}^{2}+C_{2}\right)} \\
\operatorname{SAD}_{i}=\arccos \frac{\left(\boldsymbol{D}_{i}\right)^{\mathrm{T}} \cdot \hat{\boldsymbol{D}}_{i}}{\left\|\boldsymbol{D}_{i}\right\| \cdot\left\|\hat{\boldsymbol{D}}_{i}\right\|} \\
\operatorname{MPSNR}=\frac{1}{b} \sum_{i=1}^{b} \operatorname{PSNR}_{i} \\
\operatorname{MSSIM}=\frac{1}{b} \sum_{i=1}^{b} \operatorname{SSIM}_{i}
\end{gathered}
$$

$$
\mathrm{MSAD}=\frac{1}{m n} \sum_{i=1}^{m n} \frac{180}{\pi} \mathrm{SAD}_{i}
$$

其中, $m$ 和 $n$ 分别为图像的 2 个空间维度; $b$ 为光谱 维度; $\boldsymbol{A}_{i}$ 和 $\boldsymbol{B}_{i}$ 分别代表原始图像和修复图像的第 $i$ 个波段; $\operatorname{PSNR}_{i}$ 和 SSIM $_{i}$ 是第 $i$ 波段对应的 PSNR 值和 SSIM 值; $\mu_{A_{i}}$ 和 $\mu_{B_{i}}$ 是第 $i$ 波段像素值均值; $\sigma_{\boldsymbol{A}_{i}}^{2}$ 和 $\sigma_{\boldsymbol{B}_{i}}^{2}$ 是其方差; $\sigma_{\boldsymbol{A}_{i} \boldsymbol{B}_{i}}$ 是 $\sigma_{\boldsymbol{A}_{i}}^{2}$ 和 $\sigma_{\boldsymbol{B}_{i}}^{2}$ 的协方差, $\boldsymbol{D}_{i}$ 和 $\hat{\boldsymbol{D}}_{i}$ 分别表示原始图像和修复图像的第 $i$ 个像 元光谱曲线. 很显然, MPSNR 值和 MSSIM 值越大, 表示去噪效果越有效; 而 MSAD 越小, 表明去噪 后图像的光谱保真效果越好, 所有对比实验数值 评价结果如表 1 所示. 实验仿真的硬件为 $3.00 \mathrm{GHz}$ 的酷睿 E3-1200v5 计算机, 软件平台为 64 位 Windows 7 操作系统和 Matlab 仿真软件.

实验 $1.30 \%$ 脉冲噪声以及宽度为 $1 \sim 3$ 的条带 噪声的 Washington D C Mall 图像去噪. 图 4 为该实 验可视化对比结果, 图 5 为各个波段的 PSNR 以及 SSIM 值, 图 6 所示为波段 101 的横向平均剖面图.

从图 4 可知, 所有算法均能有效地去除噪声, 但 LRTDGS 存在较为明显的伪影, 修复效果较差; LRTV 修复的图像则存在过光滑现象, 未能恢复图 像道路部分的纹理; LLRGTV 和 LRNMF 能较好地 恢复道路的上半部分, 但其下半部分的细节仍没 能较好地恢复; LRTF-DFR 无法较好地恢复道路中 间部分, 而 HTLRMA 不仅能够去除噪声, 也能较 好地恢复图像的细节信息. 表 1 所示结果通过 3 个 评估准则定量验证了 HTLRMA 的优越性, 其中 MSSIM 结果较次优的 LRNMF 领先 0.004 ; 而 MPSNR 值更是具有 $0.67 \mathrm{~dB}$ 的优势, 同时 MSAD

\begin{tabular}{|c|c|c|c|c|c|c|c|c|c|c|c|c|}
\hline \multirow{2}{*}{ 算法 } & \multicolumn{3}{|c|}{ 实验 1} & \multicolumn{3}{|c|}{ 实验 2} & \multicolumn{3}{|c|}{ 实验 3} & \multicolumn{3}{|c|}{ 实验 4} \\
\hline & MPSNR/dB & MSSIM & MSAD & MPSNR/dB & MSSIM & MSAD & MPSNR/dB & MSSIM & MSAD & MPSNR/dB & MSSIM & MSAD \\
\hline $\operatorname{LRNMF}^{[12]}$ & 40.40 & 0.9878 & 4.19 & 39.52 & 0.9852 & 3.50 & 34.29 & 0.9577 & 6.99 & 36.85 & 0.9489 & 5.29 \\
\hline LLRGTV $^{[13]}$ & 30.94 & 0.9399 & 8.70 & 36.74 & 0.9717 & 4.93 & 30.92 & 0.9385 & 8.74 & 35.94 & 0.9378 & 5.44 \\
\hline $\operatorname{LRTDGS}^{[14]}$ & 30.77 & 0.8931 & 6.92 & 30.44 & 0.8829 & 8.74 & 30.40 & 0.8823 & 6.79 & 29.70 & 0.8593 & 9.35 \\
\hline LRTF-DFR $^{[15]}$ & 33.40 & 0.9669 & 5.03 & 32.80 & 0.9378 & 7.14 & 33.31 & 0.9653 & 5.25 & 32.70 & 0.9347 & 7.24 \\
\hline LRTV $^{[30]}$ & 34.81 & 0.9615 & 7.55 & 39.45 & 0.9637 & 10.00 & 33.61 & 0.9445 & 6.73 & 36.14 & 0.9468 & 10.04 \\
\hline HTLRMA & 41.07 & 0.9920 & 3.29 & 40.08 & 0.9880 & 3.32 & 34.98 & 0.9668 & 5.03 & 37.14 & 0.9509 & 4.56 \\
\hline
\end{tabular}
值是最小的, 说明 HTLRMA 的光谱保真性能是最 佳的. 从图 5 中可见, HTLRMA 在各波段的表现相 对稳定, 而且在大部分波段中的数值都优于其他 对比算法.

表 14 组实验下不同算法的 MPSNR, MSSIM, MSAD 值

注. 最优值以粗体显示. 


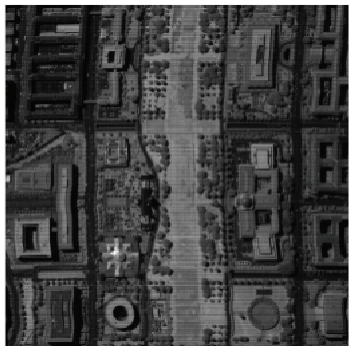

a. 原始图像

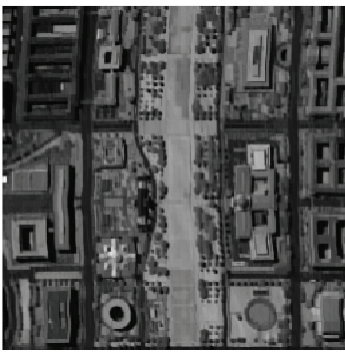

e. $\operatorname{LRTDGS}^{[14]}$

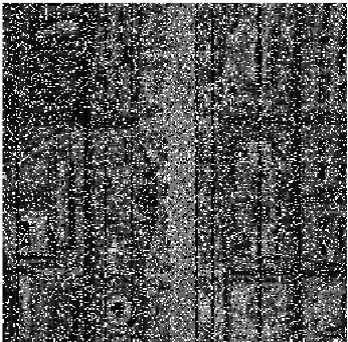

b. 含噪图像

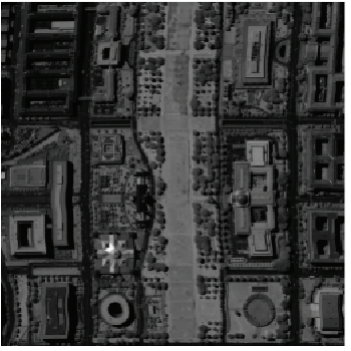

f. $\operatorname{LRNMF}^{[12]}$

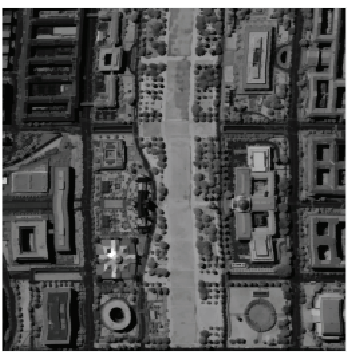

c. $\operatorname{LRTV}^{[30]}$

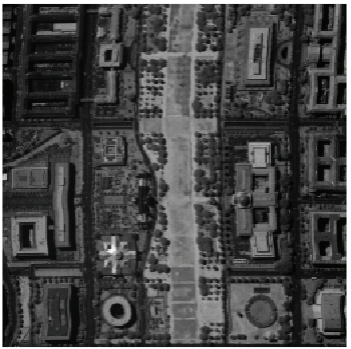

g. LRTF-DFR ${ }^{[15]}$

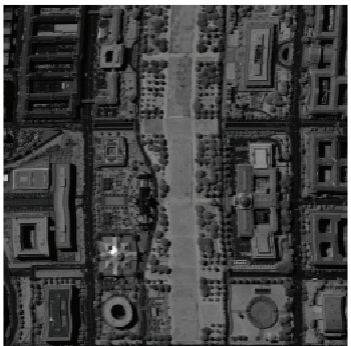

d. LLRGTV ${ }^{[13]}$

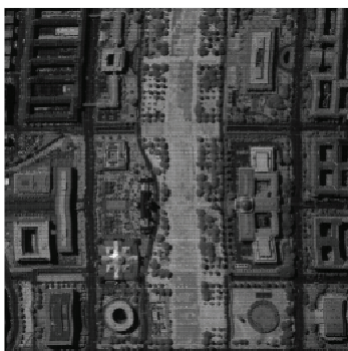

h. HTLRMA

图 4 实验 1 波段 101 去噪效果可视化对比

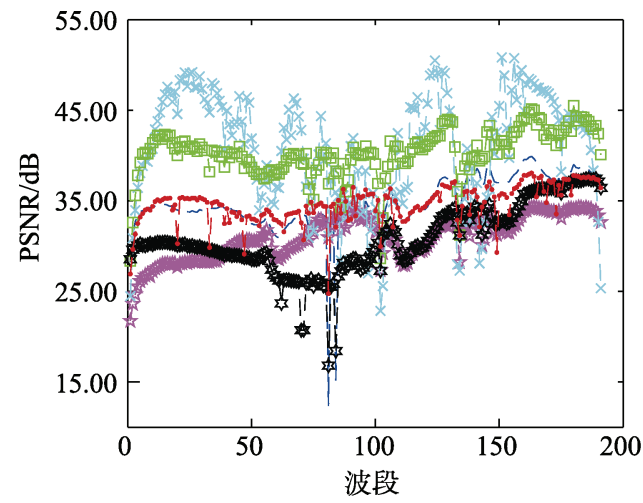

a. PSNR

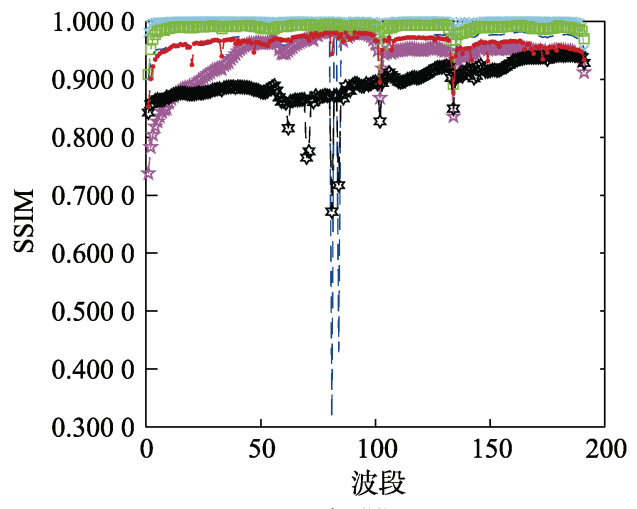

--- LRTV $^{[30]} ;-$ LLRGTV $^{[13]} ;-*-$ HTLRMA; $_{-}-$LRNMF $^{[12]} ;-$ LRTDGS $^{[14]} ;-\cdots$ LRTF-DFR $^{[15]}$

图 5 实验 1 中不同波段的 PSNR 和 SSIM 值

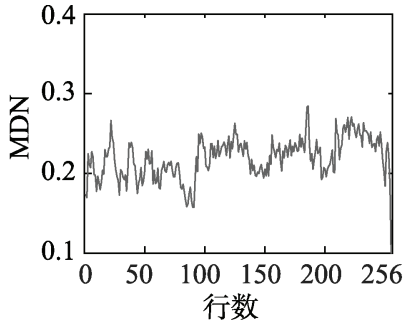

a. 原始图像

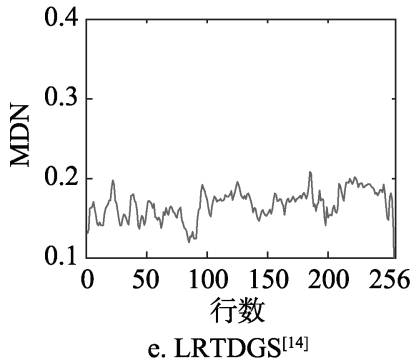

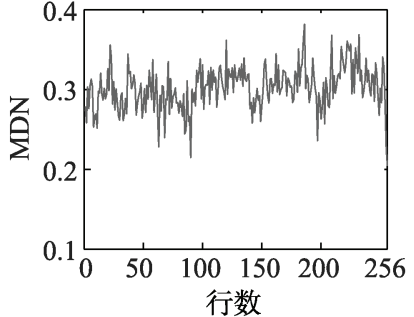

b. 含噪图像

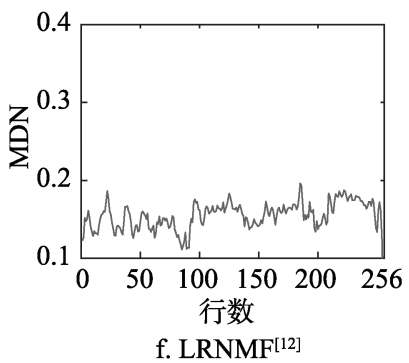

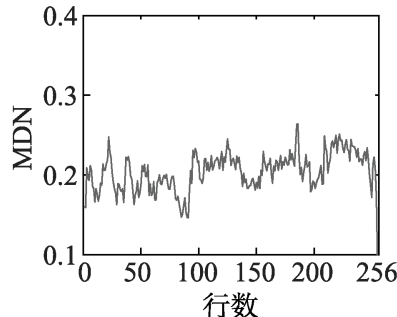

c. $\operatorname{LRTV}^{[30]}$

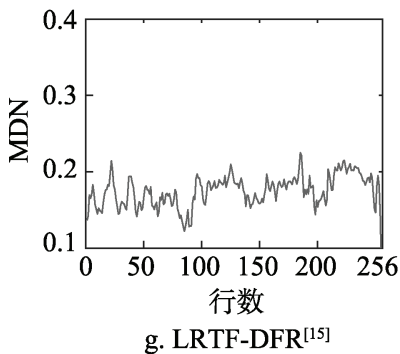

g. LRTF-DFR ${ }^{[15]}$

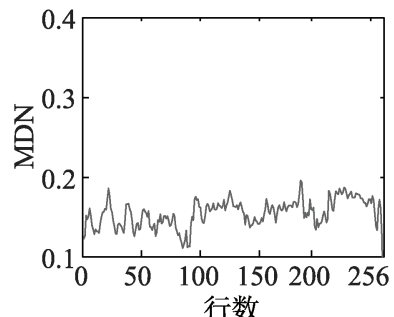

d. LLRGTV ${ }^{[13]}$

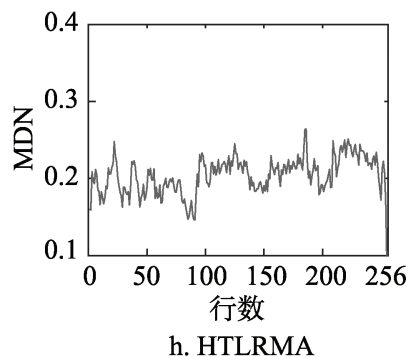

图 6 实验 1 波段 101 的横向平均剖面图 
为了进一步分析比较去噪效果, 图 6 给出了不 同去噪算法在该数据集第 101 波段的横向平均剖 面图, 该曲线图纵轴表示每行的平均数字量化值 (mean digital number, MDN), 横轴表示该波段的行 数. 从图 $6 \mathrm{~b}$ 可以看出, 由于噪声的影响, 其横向平 均剖面图出现了较为明显的波动，但所有算法修 复的图像均能获得相对光滑的 DN 曲线. 由于其他 算法修复的图像过于光滑，第 200 行的 MDN 值小 于原始图像，但 HTLRMA 的横向平均剖面图最接 近原始图像. 综上所述，本文算法不仅取得良好的 去噪效果，同时能更好地保持光谱特性.

实验 2. 对加人 $30 \%$ 脉冲噪声以及在随机波段 中加人宽度为 1 3 的条带噪声的 Pavia City Center 图像进行修复. 图 7 所示为其可视化对比结果，图 8 所示为不同波段下的 PSNR 和 SSIM 值对比，图 9
给出了 59 波段的横向平均剖面图.

从图 8 所有波段 PSNR 以及 SSIM 曲线图来看, HTLRMA 的绝大部分波段优于其他算法，展示了其 良好的性能稳定性. 值得注意的是，在波段 59 中, 所有算法的修复效果均出现了一定的下滑，从图 7 以及表 1 可知，虽然 LRTV 取得了良好的 MPSNR 以及 MSSIM 值，但波段 59 的修复效果是最差的， 其 MSAD 是所有算法中最高的, 其视觉效果以及光 谱保真效果不佳; LRTDGS 去除了大部分脉冲噪声, 但其不能较好地去除条带噪声; LRTF-DFR 也有少 量的条带噪声没有去除; LLRGTV 和 LRNMF 均能 较好地修复图像, 但细节信息处理不如本文算法. 从图 9 可以看出, 在第 150 行附近, HTLRMA 是最 光滑的，而其他算法均出现波动，这进一步表明 HTLRMA 能够较好地保证光谱的特性.

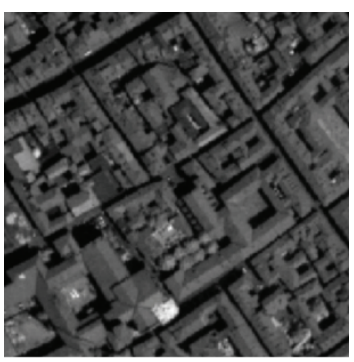

a. 原始图像

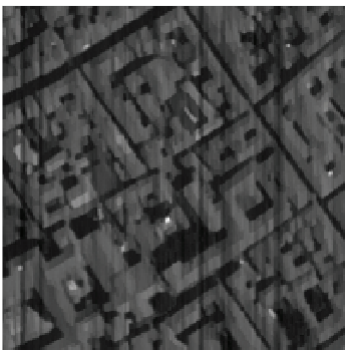

e. $\operatorname{LRTDGS}^{[14]}$

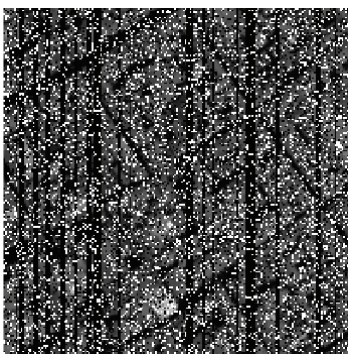

b. 含噪图像

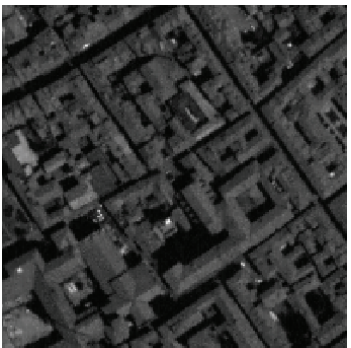

f. $\operatorname{LRNMF}^{[12]}$

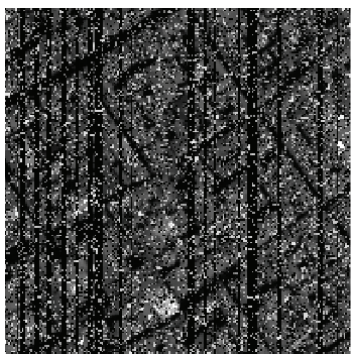

c. $\operatorname{LRTV}^{[30]}$

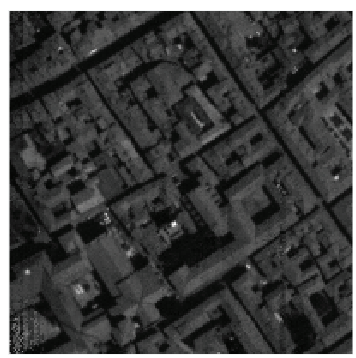

g. LRTF-DFR ${ }^{[15]}$

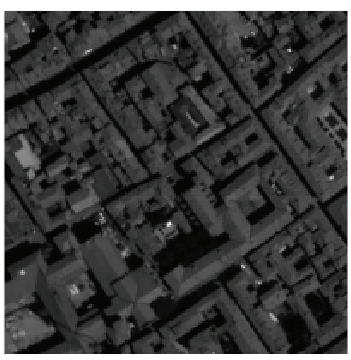

d. LLRGTV ${ }^{[13]}$

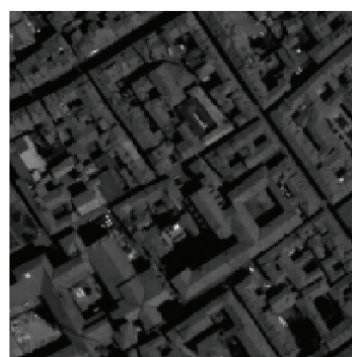

h. HTLRMA

图 7 实验 2 波段 59 去噪效果可视化对比

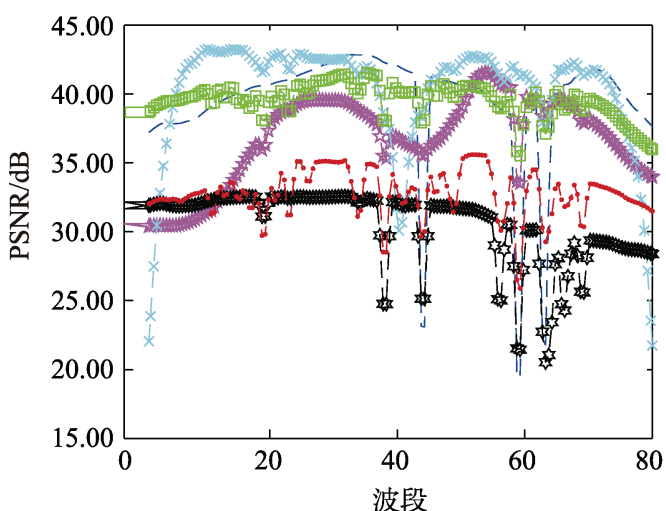

a. PSNR

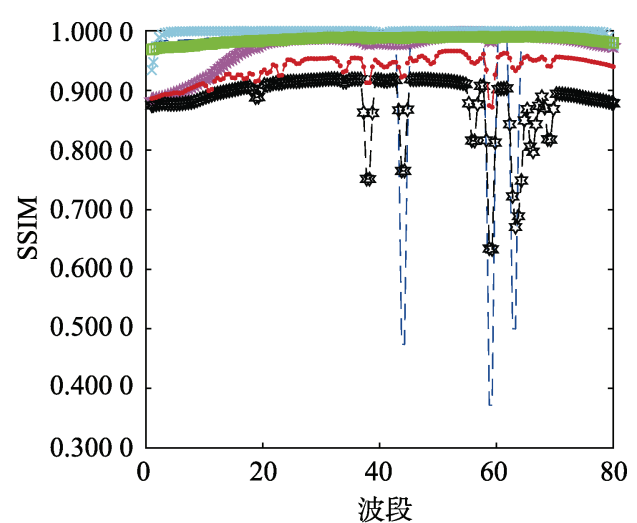

b. SSIM

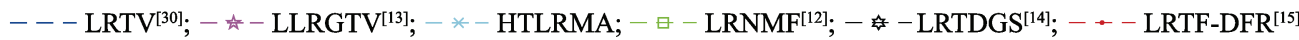

图 8 实验 2 中不同波段的 PSNR 和 SSIM 值 


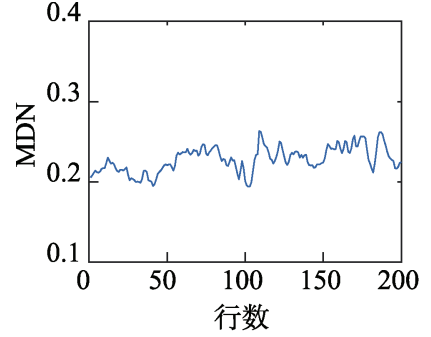

a. 原始图像

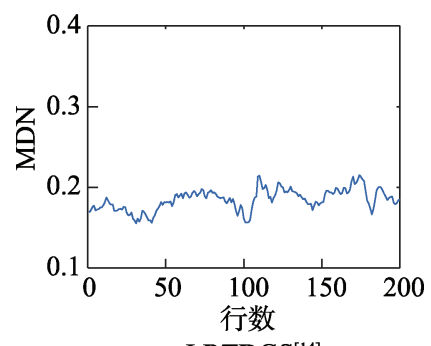

e. $\operatorname{LRTDGS}^{[14]}$

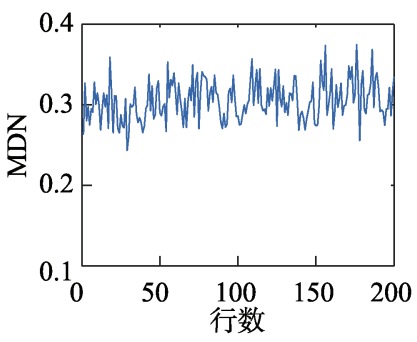

b. 含噪图像

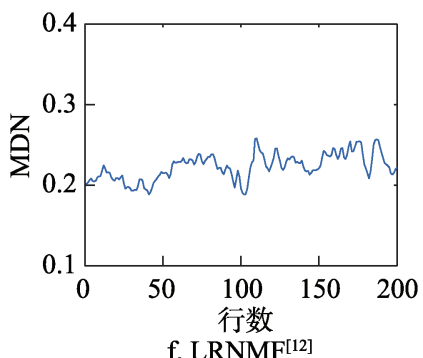

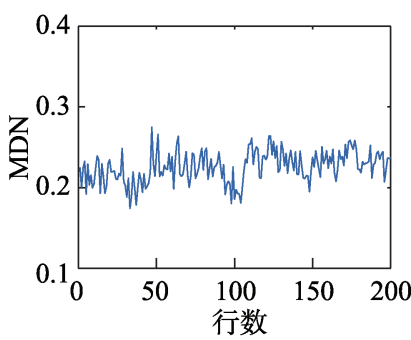

c. LRTV $^{[30]}$

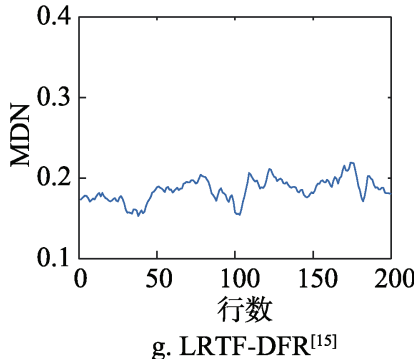

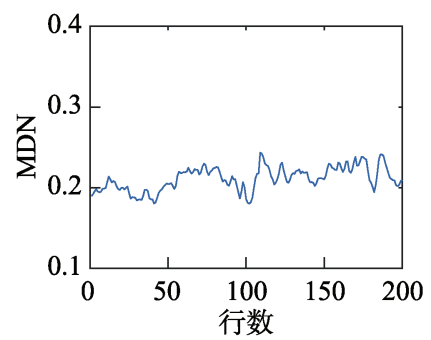

d. LLRGTV ${ }^{[13]}$

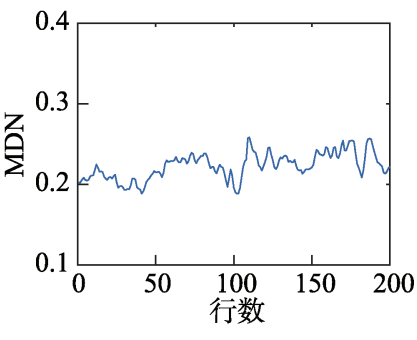

h. HTLRMA

图 9 实验 2 波段 59 的横向平均剖面图

实验 3. 对加人 $30 \%$ 的脉冲噪声、宽度为 $1 \sim 3$ 的条带噪声, 以及方差为 0.02 的高斯噪声的 Washington D C Mall 图像去噪. 图 10 显示了不同 波段下的 PSNR 和 SSIM 值.

从表 1 可以看出, HTLRMA 取得了最高的 MPSNR 以及 MSSIM 值, 展示了良好的混合噪声 去除性能. 从图 10 可知, HTLRMA 在波段 85 之前 的 PSNR 值和 SSIM 值均优于其他算法. 虽然波段 85 之后个别波段的 PSNR 值和 SSIM 值低于部分其 他算法，但整体较为平稳，而其他算法的波段间差 异非常明显, 说明了 HTLRMA 具有良好的混合噪 声去除稳定性.

实验 4. 对加人 $30 \%$ 的脉冲噪声、宽度为 $1 \sim 3$ 的条带噪声以及方差为 0.02 的高斯噪声的 Pavia City Center 图像去噪. 图 11 给出了该实验的可视化对比 结果. 图 12 显示了不同波段下的 PSNR 和 SSIM 值.

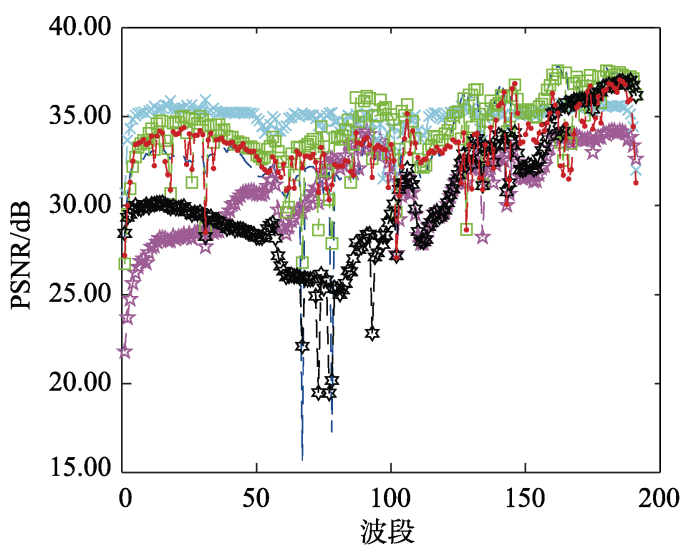

a. PSNR
从图 12 可知, HTLRMA 大部分波段的 PSNR 和 SSIM 值均领先其他算法, 除了个别波段不及 LLRGTV 以及 LRNMF, 但 HTLRMA 的稳定性优 于其他算法. 在波段 39 时, 与实验 2 类似, 所有算 法的修复性能都出现了一定的下滑, 由图 11 可知, LRTV 的修复效果的最差的, 仍存在大量的噪声; LRTDGS 去除了绝大部分的高斯和脉冲噪声, 但 仍有大量的条带噪声没有去除; LRTF-DFR 也有少 量的条带噪声存留; LRNMF 修复的图像存在部分 伪影; LLRGTV 能去除大部分噪声但细节信息不如 HTLRMA. 从表 1 可知, HTLRMA 再次取得了最高 的 MPSNR, MSSIM 以及最小的 MSAD 值, 从数据 上表明了 HTLRMA 优异的去噪以及光谱保真性能.

综上所述，本文算法去除混合噪声优于目前 最优的去噪模型, 不仅能有效地去除噪声, 也能较 好地保留图像的细节. 虽然它在某些波段修复效

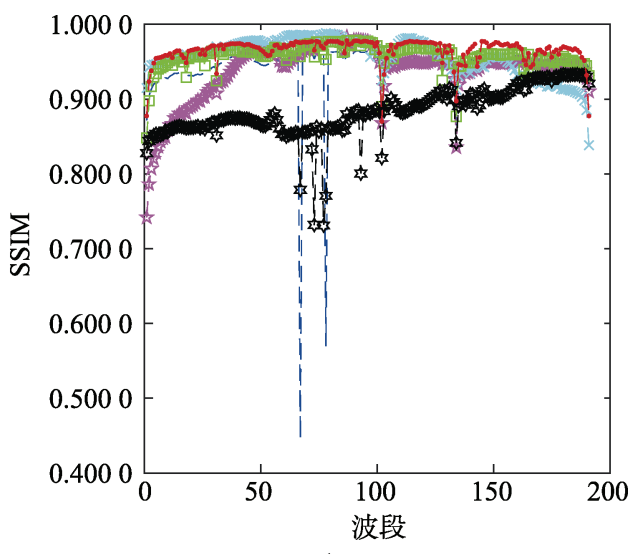

b. SSIM

--- LRTV $^{[30]} ;-$ LLRGTV $^{[13]} ;-*-$ HTLRMA; $^{2}-$ LRNMF $^{[12]} ;-$ LRTDGS $^{[14]} ;-\cdots-$ LRTF-DFR $^{[15]}$

图 10 实验 3 中不同波段的 PSNR 和 SSIM 值 


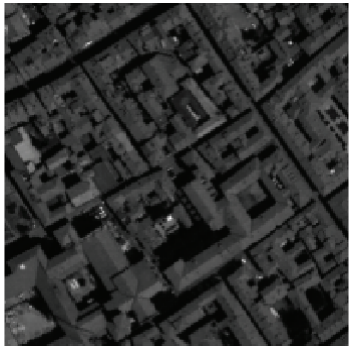

a. 原始图像

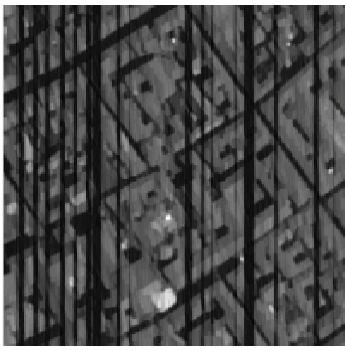

e. $\operatorname{LRTDGS}^{[14]}$

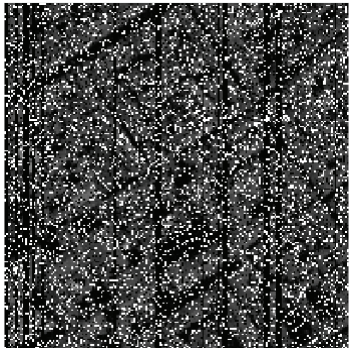

b. 含噪图像

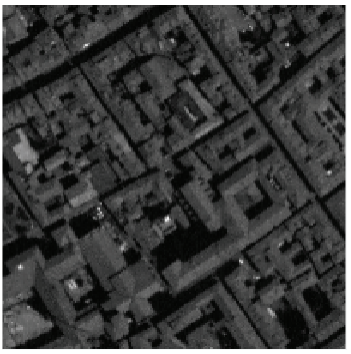

f. $\operatorname{LRNMF}^{[12]}$

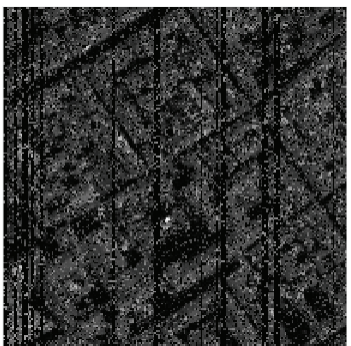

c. $\operatorname{LRTV}^{[30]}$

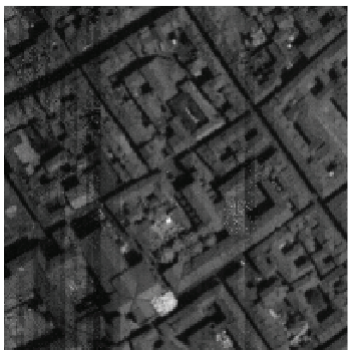

g. LRTF-DFR ${ }^{[15]}$

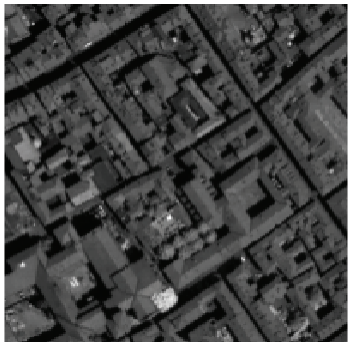

d. LLRGTV ${ }^{[13]}$

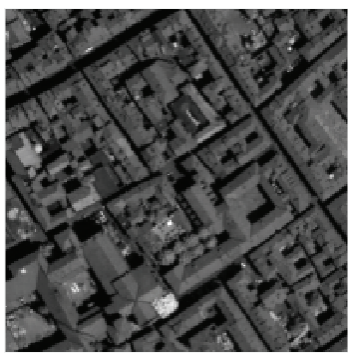

h. HTLRMA

图 11 实验 4 波段 39 去噪效果可视化对比

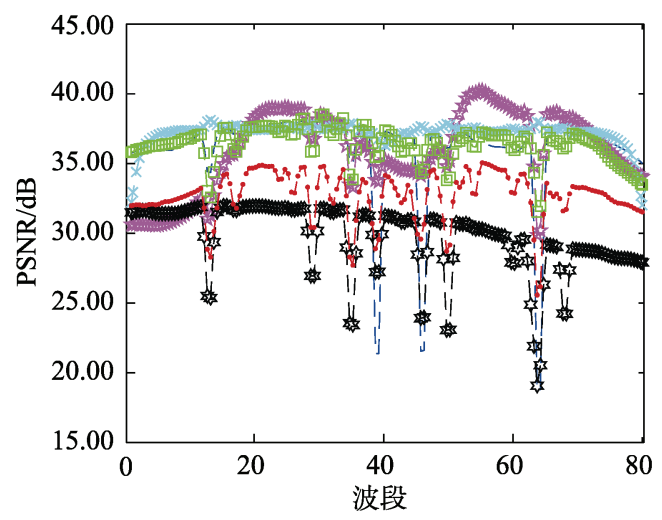

a. PSNR

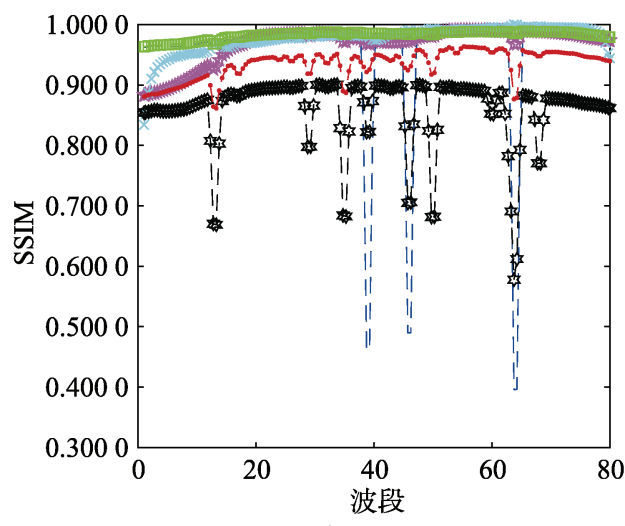

b. SSIM

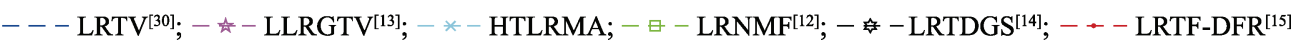

图 12 实验 4 中不同波段的 PSNR 和 SSIM 值

果不如其他算法，但能够从各波段获得相对平稳 的恢复效果，而大部分对比算法在不同波段间的 恢复差异巨大. 总体而言，本文算法对混合噪声的 综合去除效果占优.

\section{2 参数分析}

根据算法 1 和图 2 所示, HTLRMA 在应用时需 要设置 $r, \lambda$, patch 大小和步长等 4 个参数. 参数 $r$ 代表截断核范数的先验秩信息，在作者前期工作 ${ }^{[26]}$ 中已对其不同选值进行详细分析，得出在一般视 觉数据中选为 $2 \sim 15$ 的自然数具有相对稳定输出的 结论. 因此, 本文在所有实验中取 $r=5$.

正则项参数 $\lambda$ 用于平衡稀疏性和低秩性 2 个 约束项，其选值因具体应用对象不同，如何自适应 地获取其最优设定仍是一个公知问题 ${ }^{[29]}$. 一般采 用先在较大范围粗选，再通过范围缩小微调的形
式确定. 以实验 1 为例, 图 13 给出了本文算法在不 同 $\lambda$ 数值下得到的 MPSNR 结果, 其中图 13a 所示 为 $\lambda$ 取值为 $\{0.001,0.01,0.1,1,10,100\}$ 的结果, 可见 算法性能在不同 $\lambda$ 参数值时的区别较大. 选取 0.1 为基准, 进一步在 $\{0.01,0.05,0.1,0.2,0.3,0.4\}$ 范围内 微调最优 $\lambda$ 参数值如图 $13 \mathrm{~b}$ 所示, 可以发现 HTLRMA 在该范围内的性能输出较为平稳. 最终, 本文实验中选择 $\lambda=0.1$.

patch 大小是分块操作的关键参数之一, 原则 上所取的 patch 应足够精细，才能得到光滑的局部 特征, 简化零化滤波器的尺寸设置. 但是, 由于 HSI 存在复杂的纹理结构以及谱带圥余, 过小的 patch 尺寸会导致块间差异过大，无法在统一的 $\lambda$ 参数下实现整体优化. 因此, 具体 patch 尺寸大小较 难确定, 仍需要通过实验得出. 表 2 展示了本文算 
法在实验 1 中不同 patch 尺寸下的 MPSNR 结果, 从 表 2 可见, 选择 $45 \times 45$ 时取得的效果相对较好. 值得 注意的是，不同对比算法因设计标准差异，其最优 结果的 patch 尺寸设置不尽相同. 本文在实验过程中 对所有对比算法都采取了一定范围的优选预设措施, 取最佳实验结论的尺寸作为各算法的最终参数取值.

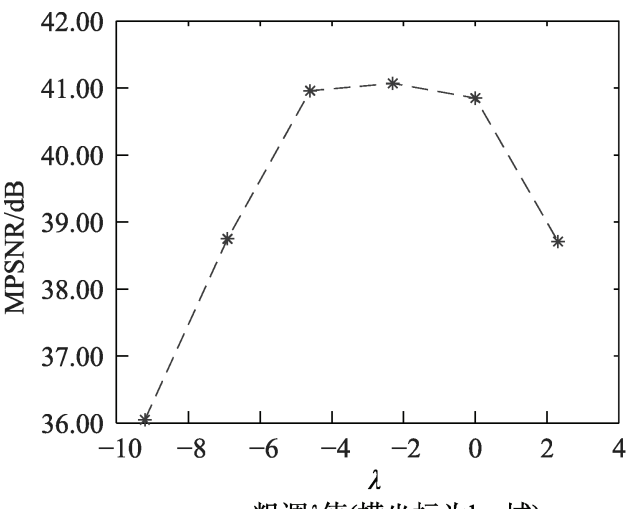

a. 粗调 $\lambda$ 值(横坐标为 $\log$ 域)

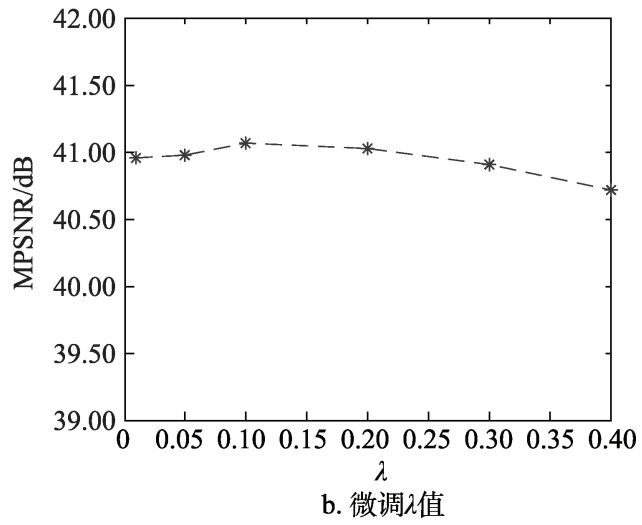

图 13 实验 1 中不同 $\lambda$ 数值下的 MPSNR 结果

表 2 实验 1 中不同 patch 尺寸下的 MPSNR 结果

\begin{tabular}{cc}
\hline patch 尺寸 & MPSNR/dB \\
\hline $25 \times 25$ & 40.17 \\
$35 \times 35$ & 40.53 \\
$45 \times 45$ & 41.07 \\
$55 \times 55$ & 41.01 \\
\hline
\end{tabular}

由于噪声分布不均匀、噪声强度不一等现象, 且图像的零化特性随着空间变化而变化, 因此为 更好地去除噪声、利用图像的零化特性以及消除 patch 轮廓等影响, 本文将图像分成重叠的 patch 来 增强可视化效果. 表 3 再次以实验 1 为例, 给出了 不同步长设定下的 MPSNR 结果. 从表 3 可见, 更 小的步长代表着更多的空间信息遍历，因此能获 得相对更优的实验效果; 相反, 更大的步长表示更 为粗略的遍历路径, 对最终恢复效果有所影响. 然 而, 更小的步长意味着更多的 patch 数量, 导致整 体图像修复效率降低. 例如, 表 3 中采用步长为 13
时获得了最高的 MPSNR 值, 但其运行时间约为 $456 \mathrm{~min}$ ，远远多于步长为 23 时所需耗费的 $135 \mathrm{~min}$. 因此, 本文为取得效率和效果的良好平衡, 在所有 实验中设定步长为 23 . 同时, 为验证算法的收玫性, 图 14 显示了随迭代次数递进的误差更新曲线, 其中 纵坐标为 $\log$ 域. 由图 14 可见, 本文优化算法随着迭 代次数的增加趋于收玫, 且收玫效率较为显著.

表 3 实验 1 中不同步长下的 MPSNR 结果

\begin{tabular}{cc}
\hline 步长 & MPSNR/dB \\
\hline 13 & 41.12 \\
23 & 41.07 \\
28 & 40.72 \\
33 & 40.41 \\
38 & 40.08 \\
\hline
\end{tabular}

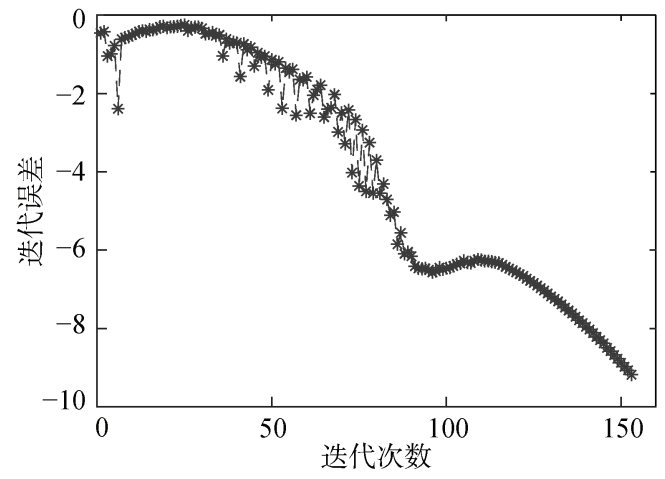

图 14 实验 1 算法收敛曲线

\section{4 结 语}

受 FRI 条件下的一维信号重构理论启发，本 文将 AF 技术扩展应用至 HSI 去噪领域. 首先, 考 虑遥感数据波段之间的相关性和空间邻域的局部 平滑性, 将输人图像进行 patch 组划分, 构建目标 优化数据子集; 其次, 针对任意 patch 子块实施 Hankel 结构化操作, 强化其低秩特性; 最后, 结合 噪声的稀疏性形成双先验约束下的目标代价函数. 所提算法兼顾了截断核范数和组稀疏范数的优越 性能，在 ADMM 优化规则下具有良好的收玫性. 实验结果也表明, 本文算法不仅在可视化效果上 优于现存高光谱混合噪声去噪模型, 而且其定量 评价指标远远高于其他竞争算法; 此外, 其在不同 波段中的恢复结果也具备更高的稳定性.

通过实际应用过程发现，受 Hankel 矩阵规模 较大的影响, 该算法的实现效率有待进一步改进. 因此, 后续工作将重点针对运行效率问题展开, 提 升算法的扩展应用能力. 


\section{参考文献(References):}

[1] Zhang Kai, Li Xuzhi. An image fusion method based on regional correlation for high resolution remote sensing images[J]. Journal of Computer-Aided Design \& Computer Graphics, 2014, 26(6): 889-895(in Chinese) (张凯，李绪志. 基于区域相关性的高分辨率遥感图像融合算 法[J].计算机辅助设计与图形学学报, 2014, 26(6): 889-895)

[2] Fang Shuai, Zhu Xiangdong, Cao Fengyun. Hyperspectral and multispectral image fusion based on unmixing-like[J]. Journal of Computer-Aided Design \& Computer Graphics, 2020, 32(1): 54-67(in Chinese)

(方帅, 朱向东, 曹风云. 基于类解混的高光谱和多光谱图 像融合算法 [J].计算机辅助设计与图形学学报, 2020, 32(1): 54-67)

[3] Elad M, Aharon M. Image denoising via sparse and redundant representations over learned dictionaries[J]. IEEE Transactions on Image Processing, 2006, 15(12): 3736-3745

[4] Dabov K, Foi A, Katkovnik V, et al. Image denoising by sparse 3-D transform-domain collaborative filtering[J]. IEEE Transactions on Image Processing, 2007, 16(8): 2080-2095

[5] Zhong P, Wang R S. Multiple-spectral-band CRFs for denoising junk bands of hyperspectral imagery[J]. IEEE Transactions on Geoscience and Remote Sensing, 2013, 51(4): 2260-2275

[6] Zhou M Y, Chen H J, Paisley J, et al. Nonparametric Bayesian dictionary learning for analysis of noisy and incomplete images [J]. IEEE Transactions on Image Processing, 2012, 21(1): 130-144

[7] Shen H F, Li X H, Zhang L P, et al. Compressed sensing-based inpainting of Aqua moderate resolution imaging spectroradiometer band 6 using adaptive spectrum-weighted sparse Bayesian dictionary learning[J]. IEEE Transactions on Geoscience and Remote Sensing, 2014, 52(2): 894-906

[8] Candès E J, Li X D, Ma Y, et al. Robust principal component analysis?[J]. Journal of the ACM, 2011, 58(3): Article No.11

[9] Xie Y, Qu Y Y, Tao D C, et al. Hyperspectral image restoration via iteratively regularized weighted Schatten $p$-norm minimization[J]. IEEE Transactions on Geoscience and Remote Sensing, 2016, 54(8): 4642-4659

[10] Lu T, Li S T, Fang L Y, et al. Spectral-spatial adaptive sparse representation for hyperspectral image denoising[J]. IEEE Transactions on Geoscience and Remote Sensing, 2016, 54(1): 373-385

[11] He W, Zhang H Y, Zhang L P, et al. Hyperspectral image denoising via noise-adjusted iterative low-rank matrix approximation[J]. IEEE Journal of Selected Topics in Applied Earth Observations and Remote Sensing, 2015, 8(6): 3050-3061

[12] Chen Y, Huang T Z, Zhao X L, et al. Hyperspectral image restoration using framelet-regularized low-rank nonnegative matrix factorization[J]. Applied Mathematical Modelling, 2018, 63: $128-147$

[13] He W, Zhang H Y, Shen H F, et al. Hyperspectral image denoising using local low-rank matrix recovery and global spatial-spectral total variation[J]. IEEE Journal of Selected Topics in Applied Earth Observations and Remote Sensing, 2018, 11(3): 713-729

[14] Chen Y, He W, Yokoya N, et al. Hyperspectral image restoration using weighted group sparsity-regularized low-rank tensor decomposition[J]. IEEE Transactions on Cybernetics, 2020, 50(8): 3556-3570
[15] Zheng Y B, Huang T Z, Zhao X L, et al. Double-factorregularized low-rank tensor factorization for mixed noise removal in hyperspectral image[J]. IEEE Transactions on Geoscience and Remote Sensing, 2020, 58(12): 8450-8464

[16] Ongie G, Jacob M. Off-the-grid recovery of piecewise constant images from few Fourier samples[J]. SIAM Journal on Imaging Sciences, 2016, 9(3): 1004-1041

[17] Vetterli M, Marziliano P, Blu T. Sampling signals with finite rate of innovation[J]. IEEE Transactions on Signal Processing, 2002, 50(6): 1417-1428

[18] Ongie G, Jacob M. A fast algorithm for convolutional structured low-rank matrix recovery[J]. IEEE Transactions on Computational Imaging, 2017, 3(4): 535-550

[19] Haldar J P. Low-rank modeling of local $k$-space neighborhoods (LORAKS) for constrained MRI[J]. IEEE Transactions on Medical Imaging, 2014, 33(3): 668-681

[20] Jin K H, Ye J C. Annihilating filter-based low-rank Hankel matrix approach for image inpainting[J]. IEEE Transactions on Image Processing, 2015, 24(11): 3498-3511

[21] Yokota T, Erem B, Guler S, et al. Missing slice recovery for tensors using a low-rank model in embedded space[C] // Proceedings of the IEEE/CVF Conference on Computer Vision and Pattern Recognition. Los Alamitos: IEEE Computer Society Press, 2018: 8251-8259

[22] Jabbarzadeh M R, Sharifi M H. Operators whose ranges are contained in the null space of conditional expectations[J]. Mathematische Nachrichten, 2019, 292(11): 2427-2440

[23] Shen H P, Huang J Z. Sparse principal component analysis via regularized low rank matrix approximation[J]. Journal of Multivariate Analysis, 2008, 99(6): 1015-1034

[24] Zheng W M, Lu C, Lin Z C, et al. $l_{1}$-Norm heteroscedastic discriminant analysis under mixture of gaussian distributions[J]. IEEE Transactions on Neural Networks and Learning Systems, 2019, 30(10): 2898-2915

[25] Cai J F, Candès E J, Shen Z W. A singular value thresholding algorithm for matrix completion[J]. SIAM Journal on Optimization, 2010, 20(4): 1956-1982

[26] Zheng J W, Qin M J, Zhou X L, et al. Efficient implementation of truncated reweighting low-rank matrix approximation[J]. IEEE Transactions on Industrial Informatics, 2020, 16(1): 488-500

[27] Rockafellar R T. Augmented Lagrange multiplier functions and duality in nonconvex programming[J]. SIAM Journal on Control, 1974, 12(2): 268-285

[28] Oh T H, Tai Y W, Bazin J C, et al. Partial sum minimization of singular values in robust PCA: Algorithm and applications $[\mathrm{J}]$. IEEE Transactions on Pattern Analysis and Machine Intelligence, 2016, 38(4): 744-758

[29] Zheng J W, Yang P, Yang X, et al. Truncated low-rank and total $p$ variation vonstrained color image completion and its moreau approximation algorithm[J]. IEEE Transactions on Image Processing, 2020, 29: 7861-7874

[30] He W, Zhang H Y, Zhang L P, et al. Total-variation-regularized low-rank matrix factorization for hyperspectral image restoration[J]. IEEE Transactions on Geoscience and Remote Sensing, 2015, 54(1): 178-188

[31] Wang Z, Bovik A C, Sheikh H R, et al. Image quality assessment: from error visibility to structural similarity[J]. IEEE Transactions on Image Processing, 2004, 13(4): 600-612 